\title{
Cisplatin-loaded core cross-linked micelles: comparative pharmacokinetics, antitumor activity, and toxicity in mice
}

This article was published in the following Dove Press journal:

International Journal of Nanomedicine

19 May 2012

Number of times this article has been viewed

\author{
Hardeep S Oberoi' \\ Natalia $\bigvee$ Nukolova ${ }^{1,2}$ \\ Frederic C Laquer ${ }^{3}$ \\ Larisa Y Poluektova ${ }^{4}$ \\ Jiangeng Huang' \\ Yazen Alnouti' \\ Masanao Yokohira ${ }^{5}$ \\ Lora L Arnold ${ }^{5}$ \\ Alexander $\mathrm{V}$ Kabanov ${ }^{1,2}$ \\ Samuel M Cohen ${ }^{5}$ \\ Tatiana K Bronich ${ }^{1,2}$ \\ 'Department of Pharmaceutical \\ Sciences and Center for Drug \\ Delivery and Nanomedicine, College \\ of Pharmacy, University of Nebraska \\ Medical Center, Omaha, NE, USA; \\ ${ }^{2}$ Department of Chemistry, MV \\ Lomonosov Moscow State University, \\ Leninskie Gory, Moscow, Russia; \\ ${ }^{3}$ Department of Chemistry, University \\ of Nebraska at Omaha, ${ }^{4}$ Department \\ of Pharmacology and Experimental \\ Neuroscience, University of Nebraska \\ Medical Center, ${ }^{5}$ Department \\ of Pathology and Microbiology, \\ University of Nebraska Medical \\ Center, Omaha, NE, USA
}

Correspondence: Tatiana K Bronich Department of Pharmaceutical Sciences and Center for Drug Delivery and Nanomedicine, College of Pharmacy, University of Nebraska Medical Center, Omaha, NE 68198-5830, USA

Tel + I 402559935 I

Fax + I 4025599365

Email tbronich@unmc.edu

\begin{abstract}
Polymer micelles with cross-linked ionic cores are shown here to improve the therapeutic performance of the platinum-containing anticancer compound cisplatin. Biodistribution, antitumor efficacy, and toxicity of cisplatin-loaded core cross-linked micelles of poly(ethylene glycol)-b-poly(methacrylic acid) were evaluated in a mouse ovarian cancer xenograft model. Cisplatin-loaded micelles demonstrated prolonged blood circulation, increased tumor accumulation, and reduced renal exposure. Improved antitumor response relative to free drug was seen in a mouse model. Toxicity studies with cisplatin-loaded micelles indicate a significantly improved safety profile and lack of renal abnormalities typical of free cisplatin treatment. Overall, the study supports the fundamental possibility of improving the potential of platinum therapy using polymer micelle-based drug delivery.
\end{abstract}

Keywords: cross-linked micelle, cisplatin, ovarian cancer, block ionomer complex, drug delivery

\section{Introduction}

The anticancer drug cisplatin (cis-diamminedichloro-platinum(II)) has revolutionized cancer management and continues to be in widespread clinical use, especially for management of tumors of the ovary, testes, and the head and neck. ${ }^{1,2}$ However, there are major limitations associated with cisplatin therapy. The majority of patients treated with cisplatin, with the exception of testicular cancer patients, experience only a partial antitumor response. Numerous systemic toxicities, such as neurotoxicity, ototoxicity, gastrointestinal disturbances, myelosuppression, and nephrotoxicity, prevent administration of higher cisplatin doses. ${ }^{3,4}$ The platinum drug has limited bioavailability due to its extensive binding to plasma proteins, spontaneous degradation in the bloodstream, and rapid clearance from the blood by glomerular excretion. ${ }^{5}$ In addition, the emergence of acquired resistance to the drug further undermines its curative potential. ${ }^{6}$ Hence, improved pharmacological specificity for this indispensible anticancer agent is much needed.

Carrier-based delivery of platinum complexes has been investigated widely over the past decade and has demonstrated its ability to improve platinum drug performance., ${ }^{7,8}$ Polymer micelles based on block ionomer complexes, in particular, are one such class of drug carriers that offers several key benefits over other colloidal systems for platinum drug delivery. These micelles, formed from copolymers containing an ionic block, can incorporate platinum complexes at an unusually high loading capacity (exceeding $30 \%$ by weight) through formation of polymer-metal complex cores. ${ }^{9}, 10$ 
Their unique advantages also include ease of preparation and drug loading, and the ability to change block copolymer chemical composition, total molecular weight, and block length ratios, permitting precise control over micelle size and morphology. ${ }^{11}$ Furthermore, readily available functional groups in the ionic block can be used for simple and controlled core cross-linking, which is essential for their structural reinforcement and stability against disintegration. ${ }^{9}$ Fixation of micelle structures by cross-linking is of interest for a number of reasons. Conventional micelles exist in solution only above the copolymer critical micelle concentration (CMC; critical association concentration for block ionomer complexes) and are therefore subject to dissociation upon dilution; for example, upon administration into the blood stream. ${ }^{12}$ Cross-linked micelles (cl-micelles) are stable at concentrations below the $\mathrm{CMC}$, can be lyophilized and redissolved as stable nanoparticles, and are less likely to collapse in response to environmental changes such as after systemic administration. ${ }^{12}$ As a result, cross-linking has the potential to increase circulation times and provide a means for temporal control over drug disposition. With respect to the material, these micelles differ from conventional micelles in behaving as nanogels, as they are composed of water-soluble polymer chains cross-linked within a nanoscale volume. Furthermore, these micelles have neither a dense core nor a defined surface and can undergo dramatic volume transitions upon environmental change. As a result, they have an unprecedented capacity for steric stabilization and decreased nonspecific interactions. The utility of such core cross-linked (cl)-micelles for encapsulation of anticancer drugs such as cisplatin ${ }^{9,13,14}$ and doxorubicin ${ }^{15,16}$ has been actively pursued by our group.

Here, we report the in vivo biodistribution, pharmacokinetics, antitumor effect, and toxicity of such cisplatin-loaded core cl-micelles (cisplatin/cl-micelles) in a mouse model, demonstrating the prolonged blood circulation, increased tumor accumulation, enhanced antitumor activity, and reduced toxicity of cisplatin/cl-micelles relative to free cisplatin.

\section{Materials and methods \\ Materials}

Poly(ethylene glycol)-b-poly(methacrylic acid) (PEG-bPMA) diblock copolymer (Mw/Mn $=1.45$, block lengths of 170 units for PEG and 180 units for PMA) was from Polymer Source Inc (Montreal, QC, Canada). Cisplatin and platinum standard for inductively coupled plasma mass spectrometry (ICP-MS) calibration were from Acros Organics (Geel, Belgium). The chemicals 1,2-ethylenediamine
(ED), 1-(3-dimethylaminopropyl)-3-ethylcarbodiimide hydrochloride (EDC), $\alpha$-D-glucose (dextrose), and others were from Sigma-Aldrich (St Louis, MO). Cisplatin for injection $\left(\right.$ Platinol $^{\circledR}$ ) was from Bristol Laboratories (Princeton, NJ). Iridium chloride internal standard for ICP-MS was from Spex Certiprep (Metuchen, NJ). Double distilled nitric acid $70 \%$ and hydrochloric acid $6 \mathrm{M}$ were from GFS chemicals (Columbus, OH). Externally threaded $2 \mathrm{~mL}$ cryovials for plasma/tissue digestion were from Corning (Lowell, MA). Heparinized tubes for blood collection (Microtainer ${ }^{\circledR}$ tubes) were from BD (Franklin Lakes, NJ). A2780 human ovarian carcinoma cells were provided by Dr P Rogers (Institute of Cancer Research, University of Bristol, UK).

\section{General procedure for synthesis of cl-micelles}

The general procedure for synthesis of PEG-b-PMA cl-micelles has been described earlier. ${ }^{9}$ Briefly, PEG-b$\mathrm{PMA} / \mathrm{Ca}^{2+}$ complexes were prepared by mixing an aqueous solution of PEG-b-PMA with a solution of $\mathrm{CaCl}_{2}$ at a molar ratio of $\left[\mathrm{Ca}^{2+}\right] /\left[\mathrm{COO}^{-}\right]=1.3$. Chains were cross-linked overnight at room temperature using ED and EDC. Extent of cross-linking was targeted to $20 \%$ and was controlled by the ratio of amine functional groups of ED to carboxylic acid groups of polymer chains. After completion of the reaction, a 1.5 molar excess amount of ethylenediaminetetraacetic acid relative to $\mathrm{Ca}^{2+}$ was added and followed with extensive dialysis, first against $0.5 \%$ aqueous ammonia and then against distilled water to remove $\mathrm{Ca}^{2+}$ ions and by products of the cross-linking reaction.

\section{Preparation of cisplatin/cl-micelles}

Cl-micelles were mixed with cisplatin $(1 \mathrm{mg} / \mathrm{mL})$ at $\mathrm{pH} 9.0$ ([cisplatin $] /[\mathrm{COOH}]=0.5)$ followed by incubation at $37^{\circ} \mathrm{C}$ for 48 hours. Unbound cisplatin was removed by ultrafiltration using Centricon ${ }^{\circledR}$ Plus-20 centrifugal filter units (molecular weight cutoff 30,000 Da; Millipore, Billerica, MA). The resultant cisplatin/cl-micelle solution was stored in the dark at room temperature until use.

\section{Particle characterization}

Particles were characterized by dynamic light scattering,

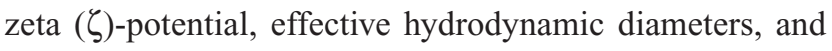
polydispersity indices as described previously. ${ }^{17}$

\section{Animal facility and standard of care}

Upon arrival, animals were placed in a facility accredited by the Association for Assessment and Accreditation of Laboratory 
Animal Care. Food and reverse osmosis water were available ad libitum throughout the study. Euthanasia was performed by $\mathrm{CO}_{2}$ asphyxiation. Treatments were administered by tail vein injection. Drug amount was calculated based on the average animal body weight. All animal protocols were approved by the University of Nebraska Medical Center Institutional Animal Care and Use Committee.

\section{Biodistribution of cisplatin/cl-micelles in mice}

Four-week-old female athymic (Ncr-nu/nu) mice were purchased from the National Cancer Institute (Bethesda, MD) and quarantined for 7 days prior to tumor inoculation. Human ovarian tumor xenografts were initiated by injecting A2780 cells $\left(5 \times 10^{6}\right.$ cells/site $)$ subcutaneously into the flanks, one above each limb. When tumor size was approximately $200-300 \mathrm{~mm}^{3}$, animals were randomized based on tumor volume into 2 treatment groups, and given either free cisplatin or cisplatin/cl-micelles at an equivalent dose of $7.5 \mathrm{mg}$-cisplatin/ $\mathrm{kg}$ body weight through intravenous tail vein injection. Animals in groups of six $(n=6)$ were euthanized at each time point of 0,5 , and 40 minutes and 1, 6, 24, 48, and 72 hours, and blood was collected in heparinized vials through cardiac puncture. Animals were then perfused with about $30 \mathrm{~mL}$ saline. Lungs, liver, kidney, spleen and tumors were excised, washed with saline and frozen over dry ice before storage at $-80^{\circ} \mathrm{C}$. Blood was immediately centrifuged at $10,000 \mathrm{rpm}$ for 15 minutes to obtain plasma, and frozen at $-80^{\circ} \mathrm{C}$ prior to further analysis. The percentage of injected dose in plasma was calculated considering plasma volume in mice to be $45.6 \mathrm{~mL} / \mathrm{kg}$. ${ }^{18}$

\section{Sample preparation and platinum measurement}

Known weights of thawed tissue were decomposed by wetashing in screw-capped vials with six volumes of concentrated nitric acid, overnight heating, and stirring at $65^{\circ} \mathrm{C}$. An iridium internal standard was added prior to digestion. Platinum concentrations were read on a Varian ICP-MS (part no 0010082601 [Walnut Creek, CA]; ICP-MS Expert software v 2.1 b103 (C) 2006) using iridium correction. Calibration range for the assay was platinum $2-100 \mathrm{ng} / \mathrm{mL}$ with extrapolation to platinum $1000 \mathrm{ng} / \mathrm{mL}$. Necessary dilutions were made if the platinum concentration exceeded the calibration range. Assay sensitivity was platinum $0.8 \mathrm{ng} / \mathrm{mL}$, with inter- and intraday assay variability not exceeding $5 \%$. However, the assay did not differentiate between micellar and nonmicellar platinum or between free and protein-bound platinum.

\section{Pharmacokinetic analysis}

Serum concentration versus time and tissue concentration versus time profiles were analyzed using noncompartmental analysis with WinNonlin 3.1 (Pharsight Corporation, Palo Alto, CA). The following parameters were determined: area under the curve (AUC), maximum platinum concentration $\left(\mathrm{C}_{\text {max }}\right)$, time to obtain maximum platinum concentration $\left(\mathrm{T}_{\max }\right)$, total body clearance $(\mathrm{Cl})$, terminal half-life of platinum $\left(\mathrm{t}_{1 / 2}\right)$, steady-state volume of distribution $\left(\mathrm{V}_{\mathrm{ss}}\right)$, the apparent terminal elimination rate constant $(\Lambda)$, and mean residence time (MRT). For serum, AUC from zero to infinity was used, whereas AUC up to 72 hours was used for tissues.

\section{Antitumor activity}

This was evaluated in female nude mice bearing subcutaneous A2780 cell ovarian xenografts. When the tumor size was approximately $100 \mathrm{~mm}^{3}$, animals were randomized based on tumor volume (three treatment groups, $n=6$ ). Mice were administered free cisplatin or cisplatin/cl-micelles at an equivalent dose of cisplatin $4 \mathrm{mg} / \mathrm{kg}$, or $5 \%$ dextrose solution through intravenous tail vein injection, a total of four times with each treatment at 4-day intervals. Animal body weight and tumor volume were monitored every second day. Tumor volume $\left(\mathrm{V}=0.5 \times \mathrm{L} \times \mathrm{W}^{2}\right)$ was estimated by measuring two orthogonal diameters (longer dimension: $\mathrm{L}$ and smaller dimension: W) of the tumor using electronic calipers. Animals were sacrificed when tumor volume exceeded $3000 \mathrm{~mm}^{3}$, greatest tumor dimension exceeded $20 \mathrm{~mm}$, tumor became necrotic, or when they exhibited a body weight loss of more than $20 \%$. All other animals were sacrificed by day 25 .

\section{Toxicity analysis}

Six-week-old female C57B1/6 mice were purchased from Charles River Breeding Laboratories (Raleigh, NC), quarantined for 7 days, and randomized based on body weight into four groups $(n=10)$. Mice were administered either free cisplatin or cisplatin/cl-micelles at an equivalent dose of cisplatin $4 \mathrm{mg} / \mathrm{kg}$, empty micelles at $2.5 \mathrm{mg} / \mathrm{kg}$, or $5 \%$ dextrose solution through intravenous tail vein injection, a total of four times with each treatment at 4-day intervals. Body weights were measured every day. Animals were monitored daily for stress.

For acute toxicity evaluation, five animals from each group were sacrificed on day 13 , one day after completion of treatment, and the remaining five animals were sacrificed at day 28. Blood, kidneys, liver, spleen, brain, spinal cord, and bone were collected at necropsy. Kidneys, liver, and spleen 
were weighed and fixed in buffered formalin. The other tissues were placed immediately in buffered formalin. Blood was collected in heparinized tubes and analyzed for plasma concentrations of blood urea nitrogen (BUN), creatinine, alkaline phosphatase, aspartate aminotransferase (AST), and alanine aminotransferase (ALT) at the Nebraska Medical Center clinical laboratory using a UniCel ${ }^{\circledR}$ DxC 600 Synchron ${ }^{\circledR}$ Clinical System (Beckman, CA).

\section{Histopathology and image analysis}

Fixed tissues were processed, sectioned, inserted into tissue cassettes, dehydrated in 70\% ethanol overnight, and paraffin embedded (UNMC Tissue Sciences Facility, Omaha, NE). Serial $5 \mu \mathrm{m}$ sections were stained with either hematoxylin and eosin (H\&E) or by immunohistochemistry (IHC). For histopathological diagnosis, H\&E-stained slides were examined by light microscopy and photomicrographs were taken using a Nikon camera mounted on a Nikon Eclipse 600 microscope (both Nikon Instruments, Melville, NY) with Adobe Elements 3.0 software (Adobe Systems, San Jose, CA). For IHC detection of cl-micelles in tissues, rabbit monoclonal antibody to PEG (anti-PEG methoxy group; Epitomics, Burlingame, CA) was used. Polymer-based HRP-conjugated anti-rabbit Dako EnVision system (Dako, Glostrup, Denmark) was used for secondary detection with 3,3'-diaminobenzidine (Dako) as the chromogen followed by counterstaining with Mayer's hematoxylin. Stained slides were analyzed using light microscopic observation and photomicrographs were obtained by Optronics (Goleta, CA) digital camera fixed to an Eclipse E800 (Nikon Instruments) using MagnaFire 2.0 software (Olympus America, Melville, NY).

\section{Flow cytometry}

Female nude mice bearing subcutaneous A2780 ovarian xenografts of approximately $800 \mathrm{~mm}^{3}$ in size were randomized into three treatment groups (with three animals per group) and administered free cisplatin, cisplatin/cl-micelles (both at cisplatin $7.5 \mathrm{mg} / \mathrm{kg}$ equivalents), or empty cl-micelles (polymer $20 \mathrm{mg} / \mathrm{kg}$ corresponding to a polymer amount equivalent to cisplatin/cl-micelles treatment). Animals were sacrificed 24 hours post-treatment. Liver, spleen, and tumors were harvested, and a part of each was used for flow cytometric analysis.

For flow cytometry, tissue samples were processed as previously described. ${ }^{19}$ Isolated viable cells $\left(1 \times 10^{6}\right)$ were stained for murine macrophages with FITC-labeled monoclonal antibody against cell surface antigen CD14 (eBioscience, San Diego, CA) and antibodies against PEG for cl-micelles (BD IntraSure kit, BD, San Jose, CA). Cells were analyzed using BD LSR II with BD FACS Diva software (BD Immunocytometry Systems, Mountain View, CA); 10,000 events were acquired in linear mode, gated to exclude debris and dead cells, and visualized in logarithmic mode. Appropriate isotype controls were used.

\section{Statistics}

For the antitumor study and toxicity studies, group means for tumor volume and body weights were evaluated using repeated measures analysis of variance with the Bonferroni post test. Survival was estimated using Kaplan-Meier analysis and compared using log-rank test. Platinum levels at various time points for biodistribution studies, blood chemistry markers for toxicity analysis, and tissue weights at sacrifice were compared using two-tailed Student's $t$-test with unequal variance. $P$ values less than 0.05 were considered significant. Analysis of variance with Bonferroni test and Kaplan-Meier analysis with log-rank test were performed using GraphPad Prism 5 (GraphPad Software, Inc, La Jolla, CA). Student's $t$-test was performed using Microsoft ${ }^{\circledR}$ Excel 2007 (Microsoft Corporation, Redmond, WA).

\section{Results}

PEG-b-PMA cl-micelles represent hydrophilic nanogel-like structures with a swollen core of cross-linked PMA networks surrounded by a PEG shell. Their synthesis, characterization, and properties have been described earlier. ${ }^{9,17}$ Briefly, their hydrodynamic diameters range from 100 to $200 \mathrm{~nm}$, have

Table I Hemolytic activity of cisplatin/cl-micelles and empty cl-micelles against human red blood cells

\begin{tabular}{lll}
\hline Treatment & $\begin{array}{l}\text { Concentration } \\
(\mu \mathrm{g} / \mathbf{m L})\end{array}$ & $\%$ hemolysis \\
\hline Empty cl-micelles & 40 & 0.00 \\
& 120 & 0.00 \\
& 400 & 0.08 \\
Cisplatin/cl-micelles & 60 & 0.00 \\
& 170 & 0.00 \\
& 500 & 0.02 \\
\hline
\end{tabular}

Notes: Whole blood from a single healthy female volunteer was collected in tubes containing Li-heparin, centrifuged at $800 \mathrm{~g}$ for 10 minutes, rinsed two times with phosphate-buffered saline (PBS), and resuspended in PBS at $37^{\circ} \mathrm{C}$. Micelles were then added to the blood cell suspension to obtain designated concentrations and a final erythrocyte concentration of $10^{8}$ cells $/ \mathrm{mL}$ (PBS, $\mathrm{pH} 7.4$ ). PBS and $1 \%$ Triton $\mathrm{X}-100$ solutions added to the blood cells samples were used as negative and positive controls, respectively. The resulting suspensions were incubated at $37^{\circ} \mathrm{C}$ for I hour under agitation of $150 \mathrm{rpm}$, centrifuged at $800 \times \mathrm{g}$ ( $10 \mathrm{~min})$ and analyzed for hemolysis in the supernatant using absorbance at $545 \mathrm{~nm}$. The percentage of hemolysis was calculated as: Hemolysis $(\%)=\left(A_{\text {sample }}-A_{0}\right) /\left(A_{100}-A_{0}\right) \times 100 \%$, where $A_{\text {sample }}$ is the sample absorbance, $A_{0}$ and $A_{100}$ are absorbance values corresponding to PBS control and to the cells completely lysed with I\% Triton X-100. Data are mean from two different samples with an internal set of duplicates. 
A

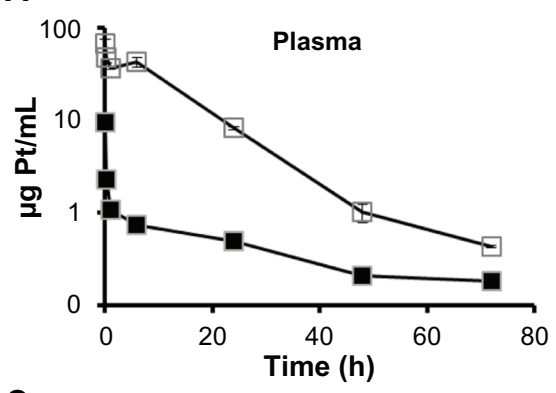

C

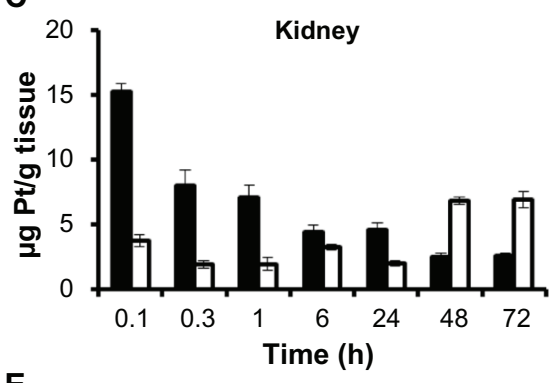

E

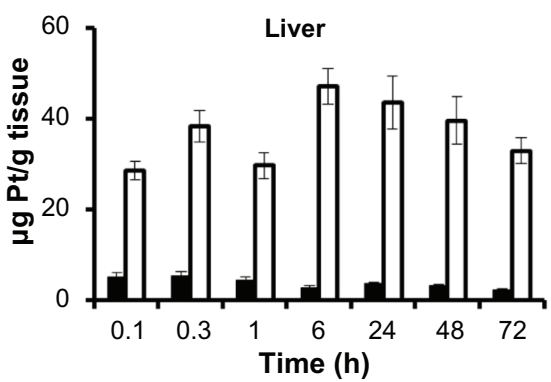

B

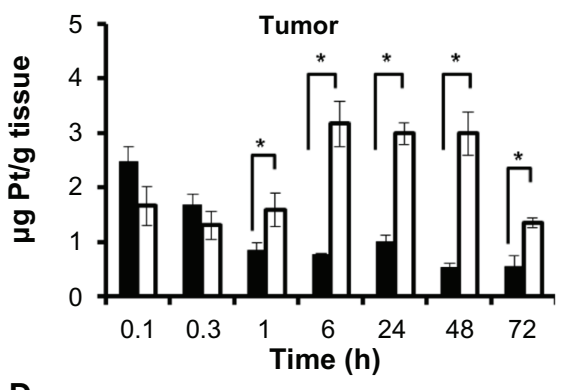

D

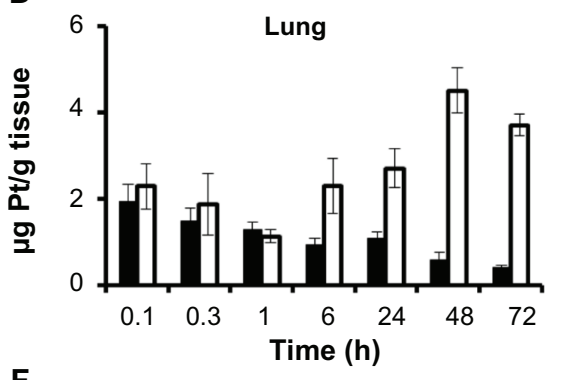

$\mathbf{F}$

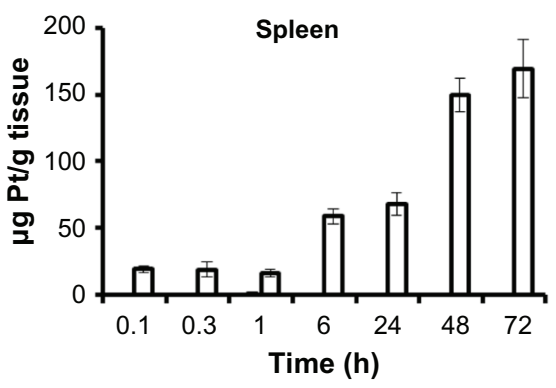

Figure I Concentration-time profile of platinum in A2780 human ovarian cancer xenograft-bearing female nude mice.

Notes: Platinum levels were measured following a single intravenous dose of cisplatin/cl-micelles ( $\square$ ) or cisplatin ( $\square)$ at $7.5 \mathrm{mg}$ cisplatin equivalents/kg body weight, in (A) plasma, (B) tumor, (C) kidney, (D) lung, (E) liver and (F) spleen. Values indicated are means \pm standard error of the mean $(n=6)$, $* P<0.05$.

a narrow size distribution (polydispersity index $<0.1$ ), and a net negative charge $(\zeta$-potential from $-20 \mathrm{mV}$ to $-30 \mathrm{mV}$ ). Cisplatin loading into cl-micelles involves a metal complex formation between PMA chains and cisplatin, and proceeds with a decrease in both size and $\zeta$-potential. ${ }^{14}$

Table 2 Pharmacokinetic parameter estimates of platinum in plasma for cisplatin or cisplatin/cl-micelle-treated A2780 human ovarian cancer xenograft-bearing female nude mice ${ }^{a}$

\begin{tabular}{lll}
\hline Parameter & Cisplatin & Cisplatin/cl-micelles \\
\hline $\mathrm{C}_{\max } \mathrm{b}(\mu \mathrm{g} / \mathrm{mL})$ & 19.22 & 80.12 \\
$\mathrm{AUC}(\mu \mathrm{g} \mathrm{h} / \mathrm{mL})$ & 32.61 & 707.03 \\
$\mathrm{Cl}(\mathrm{mL} / \mathrm{h} \mathrm{kg})$ & 2.61 & 0.14 \\
$\mathrm{~V}_{\mathrm{ss}}(\mathrm{L} / \mathrm{kg})$ & 82.48 & 1.69 \\
$\Lambda$ & 0.037 & 0.072 \\
MRT $(\mathrm{h})$ & 31.63 & 12.37 \\
\hline
\end{tabular}

Notes: ${ }^{\mathrm{T}}$ The pharmacokinetic parameters were calculated using a noncompartment model with WinNonlin program. ${ }^{b} C_{\max }$ represents the maximum observed concentration at the earliest evaluated time point of 5 min post dosage. Abbreviations: $C_{\max }$, maximum platinum concentration; $A \cup C$, area under the curve; $\mathrm{Cl}$, clearance; $\mathrm{V}_{\mathrm{ss}}$, steady-state volume of distribution; $\Lambda$, apparent terminal elimination rate constant; MRT, mean residence time.
Cisplatin/cl-micelles with a targeted cross-link density of $20 \%$ (average size of about $110 \mathrm{~nm}$, $\zeta$-potential $-12 \mathrm{mV}$, and loading capacity $30 \% \mathrm{w} / \mathrm{w}$ ) were shown previously to be optimum for a drug-release profile consistent with desirable lower release rates in systemic circulation and higher rates in the tumor environment. The released cisplatin was shown to maintain its ability to form platinum-DNA adducts, although, due to its slow release from cl-micelles, the overall in vitro cytotoxicity of the system compared with the free drug is reduced. ${ }^{14}$ Neither empty nor cisplatin-loaded cl-micelles showed any hemolytic activity toward red blood cells, an essential prerequisite for their in vivo use (Table 1). Here we evaluate the in vivo distribution, pharmacokinetics, antitumor effect, and toxicity of cisplatin/cl-micelles in mice.

\section{Pharmacokinetics and biodistribution of cisplatin/cl-micelles in mice}

Biodistribution of cisplatin loaded into cl-polymer micelles after intravenous administration was determined by 
measuring platinum concentrations in plasma and peripheral tissues at different time points up to 72 hours (Figure 1). Consistent with previous reports, ${ }^{20,21}$ free cisplatin disappeared rapidly from circulation, with only about $9 \%$ of the injected dose detectable in plasma 5 minutes after dosing. However, cisplatin/cl-micelles showed remarkably prolonged blood circulation, with more than $65 \%$ of the injected dosage retained 5 minutes after dosing and about $9 \%$ of the injected dose detectable 24 hours after dosing. Cisplatin and cisplatin/cl-micelle plasma platinum concentration versus time data was characterized by noncompartmental analysis (Table 2). Cisplatin/cl-micelletreated mice had a fourfold higher maximum observed platinum $\mathrm{C}_{\max }$, as well as 20-fold higher plasma AUC. Both $\mathrm{V}_{\mathrm{ss}}$ and $\mathrm{Cl}$ were decreased by 48-fold and 18-fold, respectively, in the cisplatin/cl-micelle treatment group compared with the cisplatin treatment group.

Peak platinum levels were observed in each tissue within 1 hour of cisplatin treatment consistent with its rapid distribution, followed by a gradual decline (Figure 1). Tumor platinum levels after cisplatin/cl-micelle treatment peaked at 6 hours, more than fourfold higher than with free cisplatin treatment $(P<0.05)$ (Figure 1B), and continued to be consistently higher up to 72 hours. At 72 hours, the last point of evaluation, tumor platinum levels remained more than twofold higher $(P<0.05)$ with cisplatin/cl-micelle treatment. Furthermore, tumor platinum $\mathrm{C}_{\max }$ was higher with cisplatin/cl-micelle treatment compared with free cisplatin treatment (Table 3 ). Consistent with previously reported literature, ${ }^{22}$ the greater part of free cisplatin appears to be excreted by kidney (Figure 1C). Indeed, being the excretory organ for free cisplatin, kidneys showed a higher platinum concentration than any other organ at all evaluated time points. Platinum concentrations in kidneys obtained from cisplatin-treated mice were highest at 1 hour after administration and gradually decreased until the end of the study. In contrast, renal levels of platinum in cisplatin/cl-micelle-treated mice remained lower than those from cisplatin-treated mice up to 24 hours, then started to increase, and did not decline until the end of the study (Figure 1C). Therefore, the renal platinum AUC following cisplatin/cl-micelle treatment was not much different from that with cisplatin treatment, although the renal platinum $\mathrm{C}_{\max }$ was much reduced (Table 3 ). This higher platinum exposure in kidneys at the late phase likely represents the released drug, consistent with in vitro release study data ${ }^{14}$ and the macromolecular nature of cisplatin/cl-micelles, which exceeds the renal filtration threshold. The liver and spleen, which are organs responsible for removing macromolecules from the bloodstream, showed a strong accumulation of cisplatin/cl-micelles (Figure 1E and F). While liver platinum levels peaked at about 6 hours after treatment and then gradually declined, spleen platinum levels continued to increase until the end of the study at 72 hours.

\section{Antitumor activity of cisplatin/cl-micelles in mice}

The in vivo antitumor efficacy was tested in mice bearing A2780 human ovarian cancer xenografts (Figure 2). Changes in relative tumor volume and body weight are shown in Figure 2A and B, respectively. Both cisplatin and cisplatin/cl-micelle treatments significantly decreased tumor burden $(P<0.05)$ compared with the control group. However, tumors in the animals treated with cisplatin/cl-micelles remained significantly smaller $(P<0.05)$ than in animals treated with free cisplatin between days 12 and 20. As a result, increased survival of the animals was observed in mice treated with cisplatin/cl-micelle compared with cisplatin alone (Figure $2 \mathrm{C}, P<0.05$ ). There was significant weight loss in the cisplatin treatment group compared with both the control and cisplatin/cl-micelle treatment groups $(P<0.05)$. In contrast, the cisplatin/cl-micelle treatment group showed no significant body weight loss compared with the control. Free cisplatin and the cisplatin/cl-micelles were injected at an equivalent dose of cisplatin $4 \mathrm{mg} / \mathrm{kg}$, determined as the maximum tolerated dose corresponding to the treatment schedule (Figure 3).

Table 3 Pharmacokinetic parameter estimates in various tissues for cisplatin or cisplatin/cl-micelle-treated A2780 human ovarian cancer xenograft-bearing female nude mice

\begin{tabular}{|c|c|c|c|c|c|c|c|c|c|c|}
\hline \multirow[t]{2}{*}{ Parameter } & \multicolumn{2}{|c|}{ Kidney } & \multicolumn{2}{|c|}{ Spleen } & \multicolumn{2}{|c|}{ Lung } & \multicolumn{2}{|c|}{ Liver } & \multicolumn{2}{|c|}{ Tumor } \\
\hline & Cis & Cis/cl-micelles & Cis & Cis/cl-micelles & Cis & Cis/cl-micelles & Cis & Cis/cl-micelles & Cis & Cis/cl-micelles \\
\hline $\mathrm{T}_{\max }(\mathrm{h})$ & 0.1 & 72.0 & 1.0 & 12.0 & 0.1 & 12.0 & 0.3 & 1.0 & 0.1 & 6.0 \\
\hline $\mathrm{C}_{\max }(\mu g / g$ tissue $)$ & 15.2 & 6.9 & 1.5 & 235.1 & 1.9 & 5.3 & 5.4 & 49.6 & 2.5 & 3.2 \\
\hline AUC ( $\mu \mathrm{g} h / g$ tissue) & 267 & 372 & 68 & 12115 & 59 & 284 & 235 & 2291 & 54 & 170 \\
\hline
\end{tabular}

Abbreviations: $\mathrm{Cis}$, cisplatin; $\mathrm{T}_{\max }$, time to reach $\mathrm{C}_{\max } ; \mathrm{C}_{\max }$, maximum platinum concentration; $\mathrm{AUC}$, area under the curve. 
A

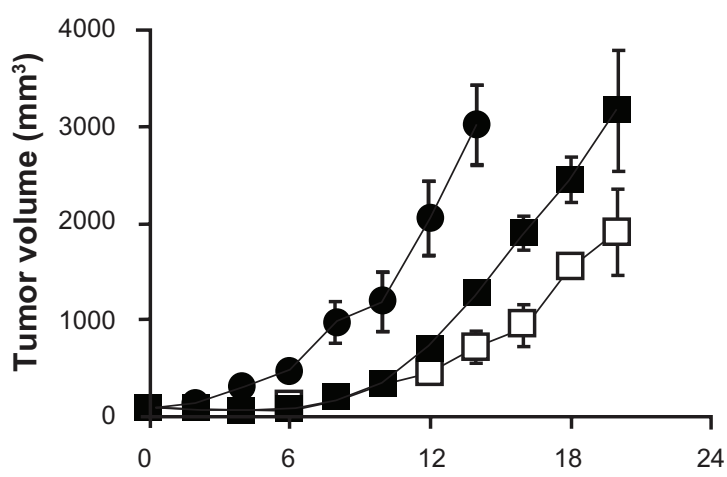

B

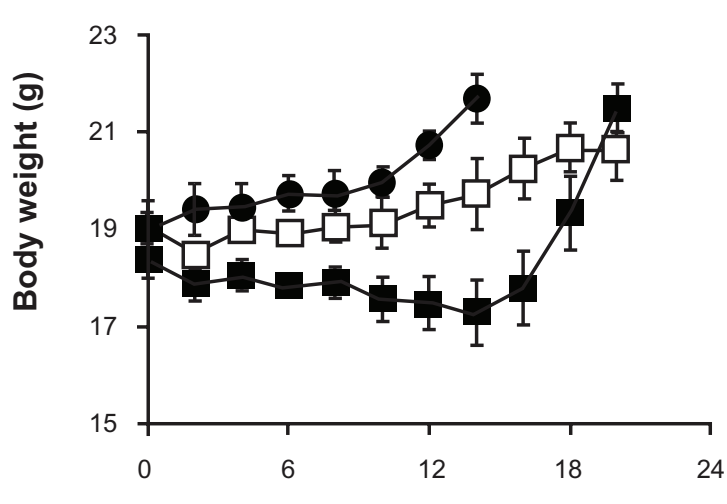

C

Day

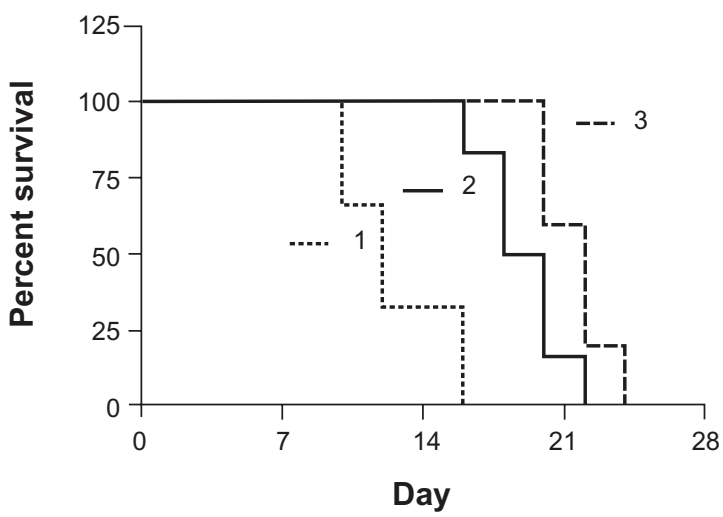

Figure 2 In vivo efficacy of cisplatin/cl-micelles in A2780 human ovarian cancer xenograft-bearing female nude mice.

Notes: Relative changes in (A) tumor volume and (B) body weight were measured following intravenous administration of cisplatin/cl-micelles $(\square)$ or cisplatin ( $\square$ ) at $4 \mathrm{mg}$ cisplatin equivalents $/ \mathrm{kg}$ body weight. Four administrations were given in total with each administration every fourth day. Control group received $100 \mu \mathrm{L}$ of $5 \%$ dextrose $(\bullet)$. Values indicated are means \pm standard error of the mean $(n=6)$. (C) Kaplan-Meier analysis of overall survival in: (I) control group, (2) cisplatin treatment group, and (3) cisplatin/cl-micelle treatment group.

\section{Toxicity analysis}

\section{Body weight analysis}

Figure 4 represents body weight changes in $\mathrm{C} 57 \mathrm{Bl} / 6$ mice during the acute toxicity study at day 13 and day 28 (Figure 4A). The mice treated with free cisplatin showed
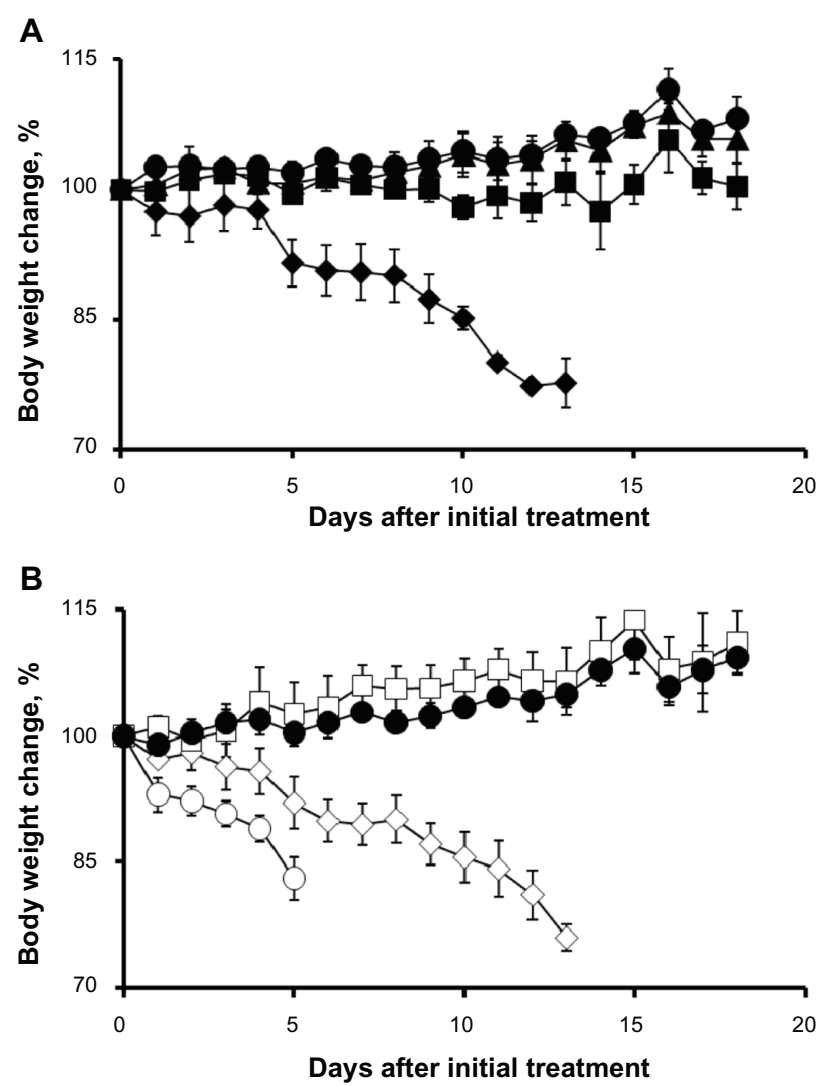

Figure 3 Relative changes in body weight in C57BI/6 mice.

Notes: (A) Cisplatin was injected intravenously every 4 days, four administrations in total, at a dose of I mg/kg ( $\mathbf{\Delta}), 3 \mathrm{mg} / \mathrm{kg}(\boldsymbol{\square})$, and $5 \mathrm{mg} / \mathrm{kg}(\bullet)$. (B) Cisplatin-loaded cl-micelles were injected at identical treatment schedule, at a cisplatin equivalent dose of $3 \mathrm{mg} / \mathrm{kg}(\square), 5 \mathrm{mg} / \mathrm{kg}(\diamond)$, and $10 \mathrm{mg} / \mathrm{kg}(O)$. Dextrose $5 \%$ treatment $(\bullet)$ was used as the control. Data are mean \pm standard error of the mean $(n=3)$. Maximum tolerated dose was assumed to be $4 \mathrm{mg} / \mathrm{kg}$ in both treatment groups since the animals lost $>20 \%$ body weight in both groups at the $5 \mathrm{mg} / \mathrm{kg}$ dose but did not lose any weight at the $3 \mathrm{mg} / \mathrm{kg}$ dose. Cisplatin-loaded cl-micelle treatment at $10 \mathrm{mg} / \mathrm{kg}$ resulted in toxic deaths in two animals. The remaining animals had $>20 \%$ weight loss and were sacrificed by day 5 .

a significant decrease in body weight compared with the control group and to the cisplatin/cl-micelle treatment group $(P<0.001)$. On day 13, the cisplatin-treated group had $>13 \%$ body weight loss compared with day 0 , significantly different from either the cisplatin/cl-micelle group or controls $(P<0.001)$. In contrast, mice treated with cisplatin/ cl-micelles showed no body weight loss although their weight gain was slower than the control group. This slower weight gain is likely due to the released cisplatin rather than the polymeric carrier itself, as animal weight change was not significantly different from that of control in the free cl-micelle treatment group.

For toxicity analysis until day 28 , the animals were allowed to recover after the fourth treatment on day 12 (Figure 4A). Animals in the empty cl-micelle treatment group and cisplatin/cl-micelle treatment group continued to gain weight, reaching an adult body weight not significantly 
A

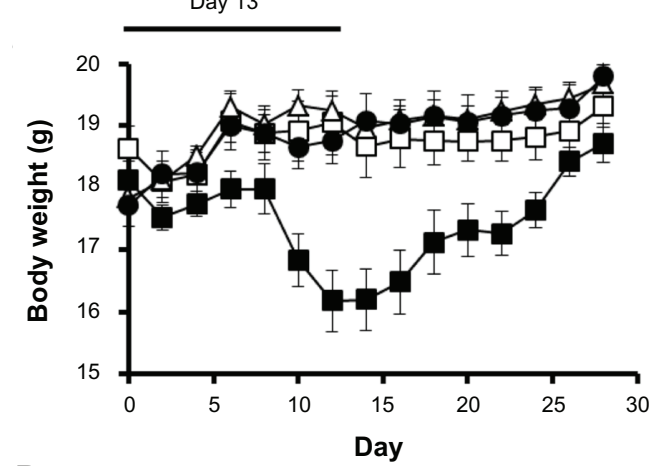

B

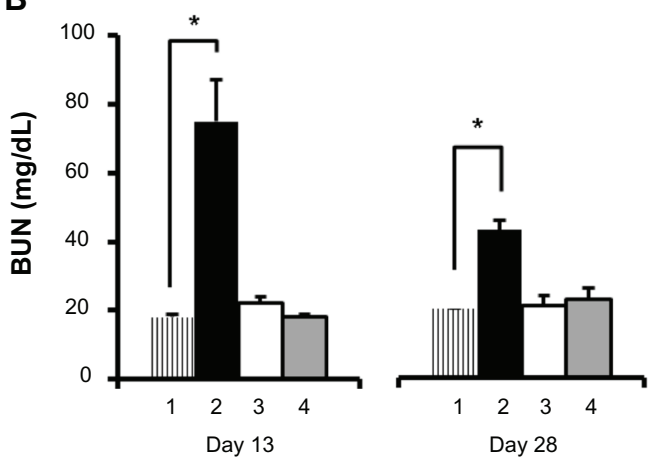

Figure 4 Acute toxicity analysis in female C57BI/6 mice.

Notes: (A) Changes in body weight $(n=5)$ until day 13 and day 28 were measured following intravenous administration of cisplatin/cl-micelles $(\square)$ or cisplatin $(\square)$ at $4 \mathrm{mg} / \mathrm{kg}$. Vehicle control groups received empty micelles $2.5 \mathrm{mg} / \mathrm{kg}(\Delta)$ or $100 \mu \mathrm{L}$ of $5 \%$ dextrose $(\bullet)$. Four administrations were given in total with each administration at every fourth day. (B) Plasma BUN concentrations $(n=3-5)$ at the end of day 13 and day 28 of toxicity analysis. Treatment groups are: (1) control, (2) cisplatin treatment, (3) cisplatin/cl-micelle treatment, and (4) empty micelle treatment. Values indicated are means \pm standard error of the mean.

Abbreviation: BUN, blood urea nitrogen.

different from controls. Mice treated with free cisplatin showed $>11 \%$ body weight loss by day 15 compared with their weight on day 0 , but then started to recover and at day 28 had an average body weight gain of approximately $3 \%$ compared with the start of treatment. However, this was still significantly less compared with the control treatment group $(P<0.001)$.

\section{Blood chemistry and tissue weights}

Plasma BUN, creatinine, alkaline phosphatase, AST, and ALT concentrations were measured at day 13 and day 28 of the study (Figure 4B and Table 4). Additionally, liver, spleen, and kidney tissue weights obtained at sacrifice and normalized to animal body weight were compared across the treatment groups (Table 5). At day13, the free cisplatin treatment group had an approximate fourfold elevation of BUN levels, significantly different from controls and the cistplatin/cl-micelle treatment group $(P<0.05)$. This increase in BUN is indicative of renal toxicity and is consistent with previously reported literature. ${ }^{4}$ Mice treated with cisplatin/cl-micelles, however, showed no significant differences in BUN levels compared to controls. Despite much higher exposure of liver and spleen to cisplatin in the cisplatin/cl-micelle treatment group (indicated by pharmacokinetics and biodistribution), there was no significant change in hepatic toxicity markers of blood chemistry (Table 4), indicating no obvious toxicity to the liver. Similarly, tissue weight analysis indicated a significant difference in kidney to body weight ratio in the cisplatin treatment group compared with the control and cisplatin/ cl-micelle treatment groups $(P<0.05)$ (Table 5). No significant differences were found in tissue to body weight ratios in cisplatin/cl-micelle treatment groups compared with the controls. At day 28, even though the animal body weights in the cisplatin-treated group had recovered, blood chemistry analysis still indicated a twofold elevation in serum BUN levels, significantly different from the cisplatin/ cl-micelle treatment group and control $(P<0.001)$ (Figure 4B). Serum BUN levels from the cisplatin/

Table 4 Clinical chemistry parameters from female C57BI/6 mice at day 13 and day 28 of toxicity analysis

\begin{tabular}{|c|c|c|c|c|c|}
\hline Treatment & $\begin{array}{l}\text { Alk phos } \\
\text { (U/L) }\end{array}$ & $\begin{array}{l}\text { ALT } \\
(\mathrm{U} / \mathrm{L})\end{array}$ & $\begin{array}{l}\text { AST } \\
\text { (U/L) }\end{array}$ & $\begin{array}{l}\text { BUN } \\
(\mathrm{mg} / \mathrm{dL})\end{array}$ & $\begin{array}{l}\text { Creatinine } \\
(\mathrm{mg} / \mathrm{dL})\end{array}$ \\
\hline \multicolumn{6}{|l|}{ Day 13} \\
\hline Control & $117 \pm 10$ & $58 \pm 17$ & $69 \pm 8$ & $18 \pm 2$ & $\mathrm{a}$ \\
\hline Cisplatin & $113 \pm 16$ & $\mathrm{a}$ & $115 \pm 24^{b}$ & $75 \pm 2 I^{b}$ & a \\
\hline Cisplatin/cl-micelles & $\mathrm{II} \pm 27$ & $43 \pm 9$ & $81 \pm 12^{c}$ & $22 \pm 4^{c}$ & $\mathrm{a}$ \\
\hline Empty cl-micelles & $128 \pm 20$ & $50 \pm 9$ & $59 \pm 7^{c}$ & $18 \pm 2^{c}$ & a \\
\hline \multicolumn{6}{|l|}{ Day 28} \\
\hline Control & $136 \pm 12$ & $85 \pm 28$ & $d$ & $20 \pm 0$ & $0.32 \pm 0.05$ \\
\hline Cisplatin & $|3| \pm 12$ & $112 \pm 45$ & $d$ & $43 \pm 6^{b}$ & $0.38 \pm 0.03$ \\
\hline Cisplatin/cl-micelles & $128 \pm 25$ & $93 \pm 20$ & $d$ & $21 \pm 5$ & $0.29 \pm 0.03$ \\
\hline Empty cl-micelles & $143 \pm 15$ & $100 \pm 43$ & $d$ & $23 \pm 5$ & $0.34 \pm 0.06$ \\
\hline
\end{tabular}

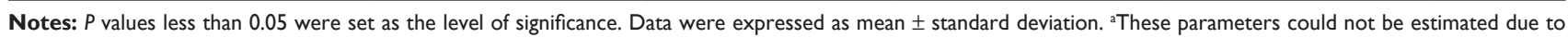
inadequate blood draw. ${ }^{b}$ Represents significant difference from controls. 'Represents significant difference from free cisplatin. ${ }^{\mathrm{d} T}$ These parameters could not be accurately measured due to hemolysis in samples at blood draw.

Abbreviations: Alk phos, alkaline phosphatase; ALT, alanine aminotransferase; AST, aspartate aminotransferase; BUN, blood urea nitrogen. 
Table 5 Tissue weights at sacrifice from female C57BI/6 mice at day I3 and day 28 of toxicity analysis

\begin{tabular}{|c|c|c|c|}
\hline Treatment & Liver/BW & Spleen/BW & Kidney/BW \\
\hline \multicolumn{4}{|l|}{ Day I3 } \\
\hline Control & $0.0505 \pm 0.0013$ & $0.0040 \pm 0.0006$ & $0.0114 \pm 0.0007$ \\
\hline Cisplatin & $0.0527 \pm 0.0012$ & $0.0028 \pm 0.0003^{a}$ & $0.0145 \pm 0.0010^{a}$ \\
\hline Cisplatin/cl-micelles & $0.0500 \pm 0.0030$ & $0.0040 \pm 0.0005$ & $0.0116 \pm 0.0005$ \\
\hline Empty cl-micelles & $0.0510 \pm 0.0033$ & $0.0040 \pm 0.0009$ & $0.0115 \pm 0.0007$ \\
\hline \multicolumn{4}{|l|}{ Day 28} \\
\hline Control & $0.047 I \pm 0.004 I$ & $0.0037 \pm 0.0005$ & $0.0117 \pm 0.0006$ \\
\hline Cisplatin & $0.0542 \pm 0.0025^{a}$ & $0.0040 \pm 0.0007$ & $0.0116 \pm 0.0005$ \\
\hline Cisplatin/cl-micelles & $0.0461 \pm 0.0048$ & $0.0038 \pm 0.0005$ & $0.0119 \pm 0.0007$ \\
\hline Empty cl-micelles & $0.0468 \pm 0.0018$ & $0.0036 \pm 0.0004$ & $0.0120 \pm 0.0008$ \\
\hline
\end{tabular}

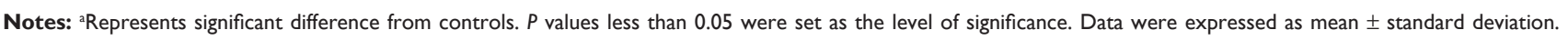
Abbreviation: BW, body weight.

cl-micelle treatment group continued to be normal, showing no long-term effects. The partial recovery in serum BUN levels in the cisplatin group reflects a reversible component of cisplatin-induced renal toxicity. ${ }^{3}$

\section{Tissue histopathology}

Light microscopic examination of H\&E stained sections from day-13 sacrificed animals (Figure 5) indicated focal subcapsular tubular vacuolization and basophilia in kidneys from all animals in the cisplatin-treated group (Figure 5B). In some animals, individual tubular necrosis, collecting duct necrosis at the tip of the papilla, and decreased cellularity in the papilla were also observed. Bone marrow suppression was the most prominent observation as indicated by hematopoietic hypocellularity and increased amounts of intercellular material (Figure 5F). In contrast, no histopathological changes were observed in kidneys (Figure 5C and D) and bone marrow (Figure 5G and $\mathrm{H}$ ) from animals in the cisplatin/cl-micelle-treated or in empty cl-micelles-treated groups compared with the control. Tissue sections from animals sacrificed at day 28 indicated partial recoveries in affected tissues (Figure 5J). Clusters of regenerating tubules were observed in the subcapsular region of the kidney cortex. However, some of the other pathological renal changes were not completely reversible at this time point as indicated by persistence of basophilic tubules related to continuing regeneration, and an interstitial lymphocytic, chronic inflammatory infiltrate. Bone marrow changes seen at day 13 returned to normal by day 28 (Figure $5 \mathrm{~N}$ ), indicating only an acute, reversible effect of cisplatin treatment. No histopathological changes were observed in analyzed tissues in the cisplatin/cl-micelle treatment group (Figure 5K and $\mathrm{O}$ ). In addition, no histopathological changes were observed by light microscopic observation of the liver and spleen from mice in the cisplatin/cl-micelle group compared with the controls, despite much higher platinum accumulation in these tissues relative to free-drug treatment (Figure 6).

Distribution of polymer micelles in tissues was further analyzed by IHC (Figure 7). No accumulation of cl-micelles was found in either the cortex or tubular regions of the kidney (cisplatin/cl-micelles: Figure 7A and B; empty cl-micelles: Figure $7 \mathrm{C}$ and D). Consistent with biodistribution data, a significant accumulation of cl-micelles was observed in spleen and liver. Liver showed a homogeneous distribution of micelles (cisplatin/cl-micelles: Figure 7E and F; empty cl-micelles: Figure $7 \mathrm{G}$ and $\mathrm{H}$ ). However, in spleen micelles were found largely in the red pulp region and were scarce to nonexistent in the white pulp region (cisplatin/cl-micelles: Figure 7I and J; empty cl-micelles: Figure 7K and L). Morphology of PEG-positive cells together with their tissue localization indicated that the majority of the cl-micelle uptake is by macrophages.

\section{Flow cytometry}

The nature of the cells involved in cisplatin/cl-micelle uptake was further investigated using flow cytometry. Cells isolated from the liver, spleen, and tumor 24 hours after treatment were stained using fluorescence-labeled antibodies against the PEG methoxy group of the block copolymer and mouse macrophage marker CD14. The relative population of $\mathrm{CD} 14+$ cells isolated from various tissues was similar amongst various treatment groups (Figure 8A). The majority of PEG+ cells (70\%-80\%) in spleen and liver also expressed CD14+ (Figure 8B). Together, these results were in accordance with IHC data discussed above, indicating macrophage capture of cisplatin/cl-micelles. Tumor tissue was found to have two 


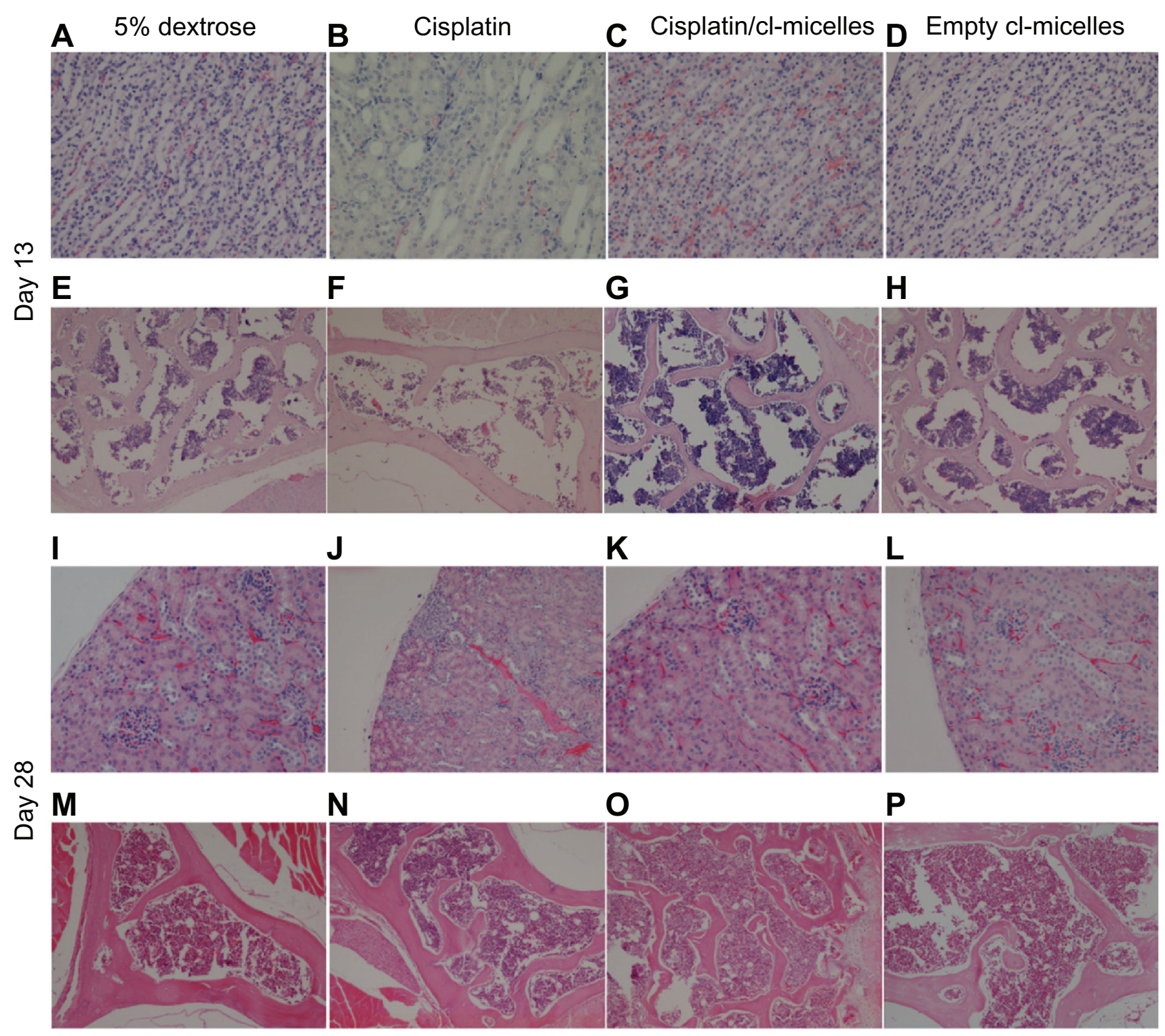

Figure 5 Histopathological changes in kidney and bone marrow tissues from toxicity analysis in female C57BI/6 mice.

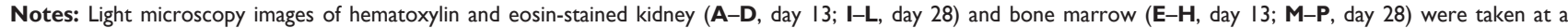
magnification of $200 \times$ and $100 \times$ respectively. Tissue samples were collected at day 13 and day 28 following intravenous administration of cisplatin/cl-micelles or cisplatin at $4 \mathrm{mg} / \mathrm{kg}$ body weight. Vehicle control groups received $2.5 \mathrm{mg}$ free micelles/kg body weight or $100 \mu \mathrm{L}$ of $5 \%$ dextrose. Four administrations were given in total, each administration performed every fourth day.

distinct populations of PEG+ cells. One of them (about $40 \%$ of total count) was also CD14+ and represents cl-micelles taken up by macrophages associated with the tumor. The remainder of cl-micelles delivered to the tumor seems to be associated with tumor cells. Comparable results were observed for the empty cl-micelle treatment group (Figure 8C).

\section{Discussion}

Polymer micelles with a cross-linked ionic core represent functional drug carriers with several key structural features suitable for effective drug delivery. These are a cross-linked ionic core, a hydrophilic PEG shell, and a nanoscale size. Properties of the cl-micelles can be altered by varying the block ionomer structure, number of the cross-links, hydrophobicity of the cross-linker, and use of cross-linkers with ionizable groups. ${ }^{23}$ Therefore, a wide variety of cl-micelles with different sizes and charges can be synthesized from the same micellar template. Rapid caveolae-mediated internalization of these cl-micelles in epithelial cancer cells but not in tight junction forming normal epithelial cells was recently reported by our group, and indicates tumor specificity of these nanosystems. ${ }^{24}$ Cisplatin, a well-known cancer chemotherapeutic agent, 

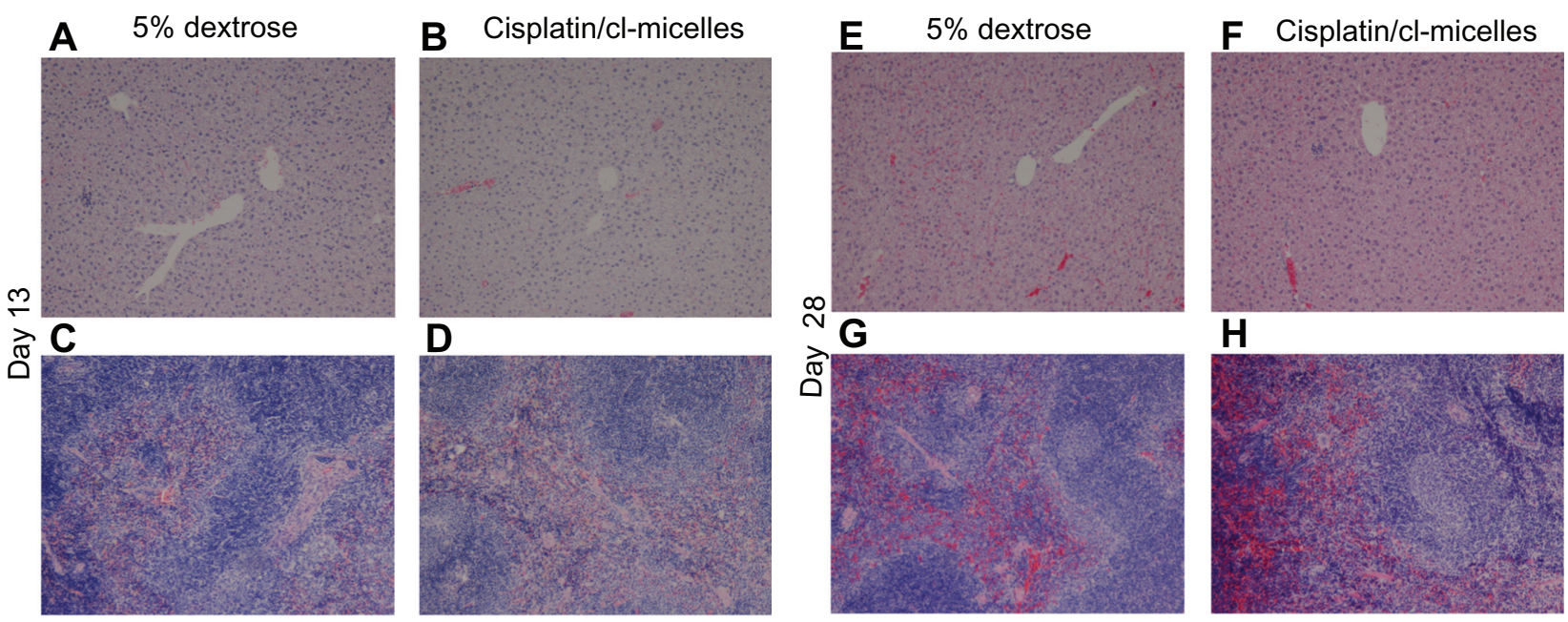

Figure 6 Light microscopy images (original magnification 100X) of hematoxylin and eosin-stained liver and spleen tissues from toxicity analysis in female C57BI/6 mice. Notes: No histopathological changes were observed in liver ( $\mathbf{A}$ and $\mathbf{B}$, day I3; E and F, day 28) and spleen (C and D, day I3; $\mathbf{G}$ and $\mathbf{H}$, day 28) following administration of free cisplatin at a dose of $4 \mathrm{mg} / \mathrm{kg}$, cisplatin-loaded cl-micelles at a cisplatin equivalent dose of $4 \mathrm{mg} / \mathrm{kg}$, or empty micelles at $2.5 \mathrm{mg}$ polymer/kg body weight. Treatment was administered by bolus intravenous tail vein injection $(n=5)$, four treatments in total, with each treatment at a 4-day interval. The animals were sacrificed using $\mathrm{CO}_{2}$ euthanasia on day 13 and day 28 for toxicity analysis. Hematoxylin and eosin staining was performed as described in materials and methods.

was incorporated into the ionic core of micelles with remarkably high efficiency, where cross-linking stabilized the micelles against structural disintegration in aqueous dispersions and prevented premature release of the drug. ${ }^{9}$ Under physiological conditions, due to the presence of abundant counter ions, the coordination bond between cisplatin and the carboxylate group of the polymer core is lost, allowing slow and sustained release of active platinum complexes. ${ }^{9}$ Interestingly, the structural determinants mentioned above allow a precise control of drug loading
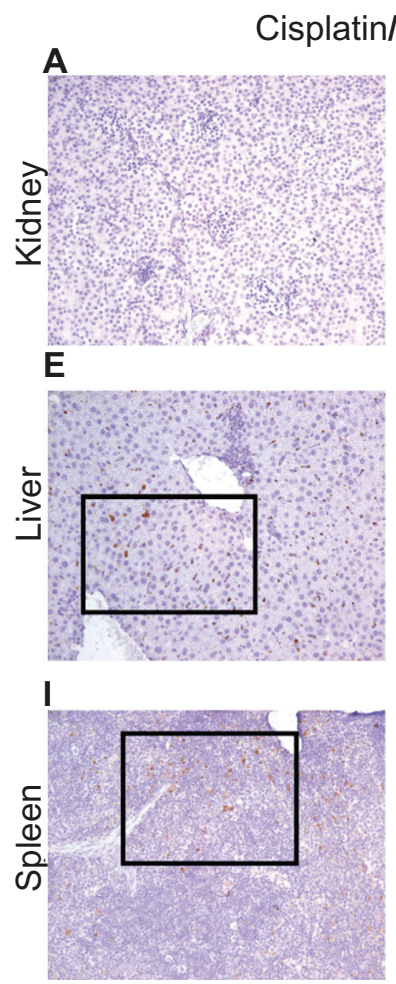

cl-micelles

B

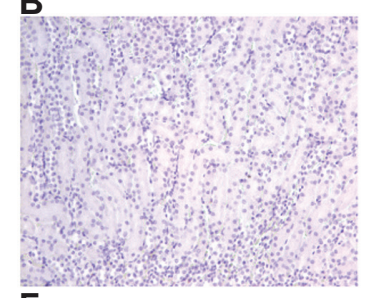

$\mathbf{F}$

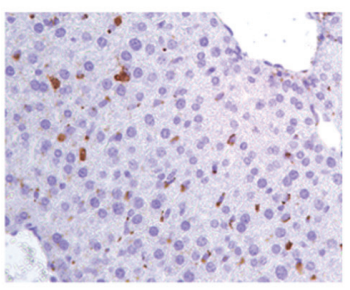

$\mathbf{J}$

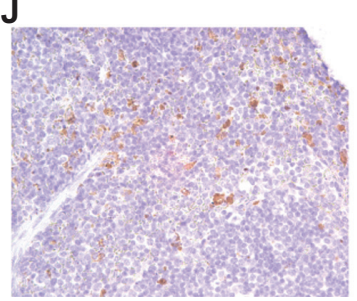

C

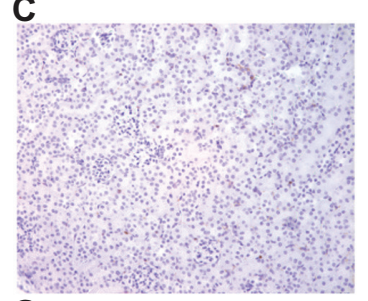

G

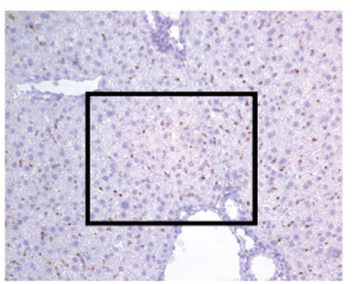

K

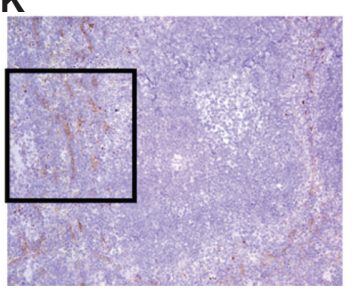

Empty cl-micelles

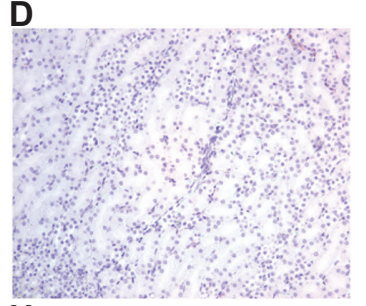

H
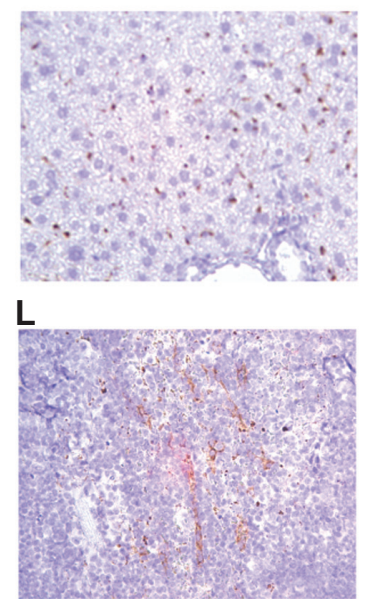

Figure 7 Immunohistochemistry images of PEG stained tissues from acute toxicity analysis in female C57BI/6 mice.

Notes: No accumulation was observed in the kidney (magnification $200 \times$ ) from either cisplatin-loaded (A, cortex; B, tubules) or empty micelles (C, cortex; D, tubules) treatment groups. Liver (E-F, cisplatin/cl-micelles; $\mathbf{G}-\mathbf{H}$, empty micelle) and spleen (I-J, cisplatin/cl-micelles; K-L, empty micelle) showed strong accumulation of micelles (magnification $200 \times / 400 \times$ ). Spleen shows accumulation of micelles only in the red pulp region. 

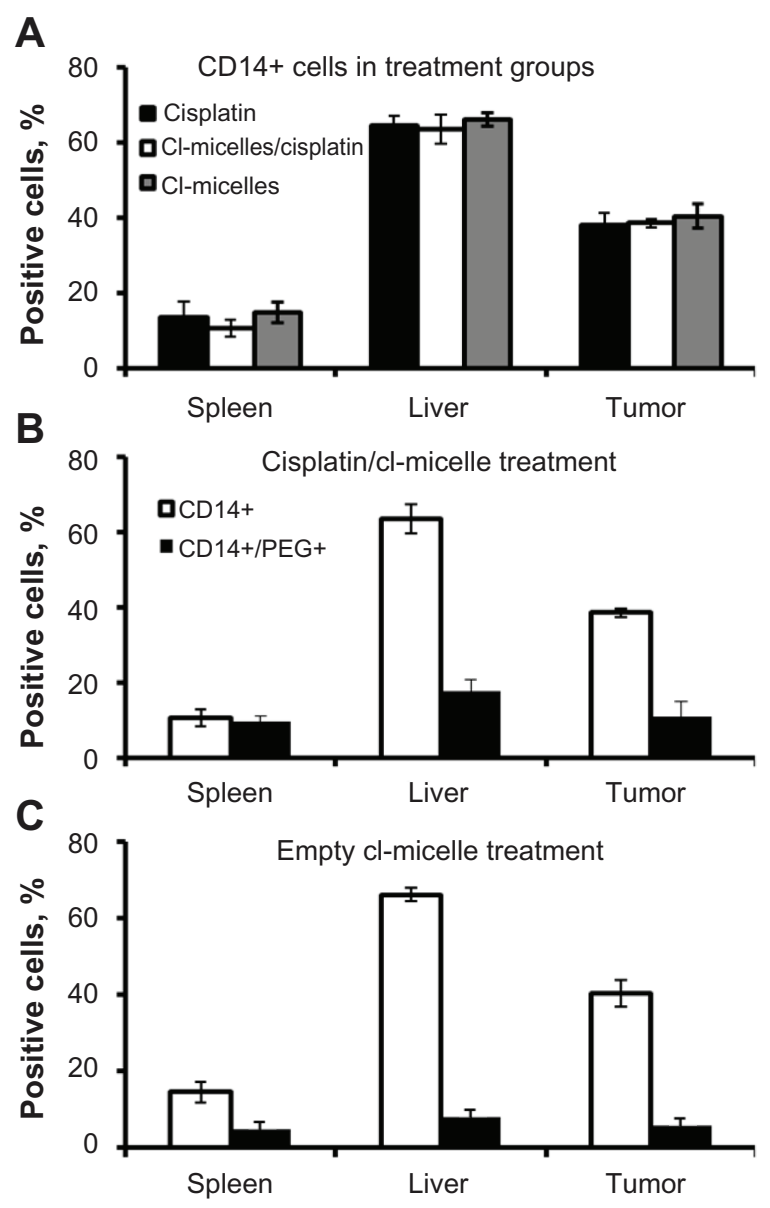

Figure 8 Uptake of cisplatin/cl-micelles by macrophages.

Notes: Female nude mice $(n=3)$ bearing A2780 ovarian cancer xenografts (approximately $800 \mathrm{~mm}^{3}$ ), administered a single intravenous tail vein injection of free cisplatin or cisplatin/cl-micelles $(7.5 \mathrm{mg} / \mathrm{kg}$ ), or empty micelles (20 mg polymer $/ \mathrm{kg}$ body weight) were sacrificed 24 hours post-treatment and liver, spleen, and tumors were harvested for flow cytometric analysis. Staining was performed with anti-CDI4 antibody and antiPEG antibody as described in material and methods. (A) Relative population of CDI4 positive cells amongst the treatment groups was similar. (B) and (C), majority of the PEG positive cells in the micelle treatment group liver and spleen costained for mouse CDI4 (70\%-80\%), indicating macrophage uptake of micelles in these organs. Tumor had two distinct populations of cells with about $30 \%-40 \%$ of PEG positive cells costaining for CDI 4 while the remaining associated with other tumor cells.

capacity, loading efficiency, and release behavior of the encapsulated drug. ${ }^{14}$

Biodistribution and therapeutic effectiveness of cisplatin/ cl-micelles were compared with those of cisplatin in solution in nude mice bearing human ovarian carcinoma A2780 xenografts. Significantly prolonged circulation of cisplatin/ cl-micelles was observed relative to free drug. In addition, cisplatin/cl-micelles had a more than 20-fold higher plasma AUC and about 50-fold lower $\mathrm{V}_{\mathrm{ss}}$ compared with cisplatin, indicating that cisplatin/cl-micelles are more localized in the plasma compartment. Free cisplatin was rapidly distributed to all major organs, with the kidney having the highest drug exposure compared with other organs at all evaluated time points. Nephrotoxicity of cisplatin was shown to be highly correlated with peak platinum concentration in kidney tissues rather than the organ AUC. ${ }^{25}$ Indeed, reduced platinum $\mathrm{C}_{\max }$ with cisplatin/cl-micelle treatment could ameliorate cisplatininduced toxicity. Free-drug treatment led to significant weight loss in animals, elevation of serum BUN levels, and histopathologic evidence of renal tubular toxicity, whereas the micelle-drug treatment group had normal markers. While bone marrow suppression occurred only during the treatment phase and was reversed by stopping the drug treatment, renal changes were more extensive and sustained, with significant changes in serum BUN, creatinine, and tissue histopathology, even at day 28 of the study. Reduced nephrotoxicity of cisplatin/cl-micelles is likely due to its macromolecular size coupled with slow release of the drug, which leads to a lower renal tubular drug exposure. Since the size of cisplatin/cl-micelle complexes (MW $>10^{6} \mathrm{Da}$ ) is far above the renal filtration threshold (approximately 40-50 kDa for hydrophilic copolymers), ${ }^{26}$ the kidney may not allow filtration of these complexes. Indeed, antibody against the PEG methoxy group of copolymers failed to recognize any micelles in the renal tubules or cortex. This is a significant result considering renal toxicity is the most severe, dose-limiting toxicity with cisplatin treatment.

A strong accumulation of cisplatin/cl-micelles was observed in liver and spleen. However, such accumulation did not lead to any toxic effects. Cisplatin/cl-micelle treated animals, even after multiple doses, had normal weight gain, no indication of stress, normal serum levels of markers of hepatic and renal toxicity, and no changes in tissue histopathology. IHC and flow cytometry analysis indicates that the PEG-positive cells in the liver and spleen were macrophages, consistent with the known reticuloendothelial capture of nanoparticles. ${ }^{27}$ The clearance of cisplatin/ cl-micelles from these tissues is expected to be largely through the hepatobiliary route, although renal elimination of released cisplatin and filterable particle fragments generated over time is also possible. Biliary clearance was indeed shown to be the dominant route for elimination of silica nanoparticles of similar size and negative $\zeta$-potential. ${ }^{28,29} \mathrm{It}$ is quite likely that cisplatin/cl-micelles carrying macrophages will undergo several rounds of apoptotic cell and recapture of apoptotic bodies by surrounding macrophages before any significant elimination takes place. Therefore, clearance is expected to be slow and is consistent with our data indicating a significantly reduced PEG staining in liver and spleen tissue 
A

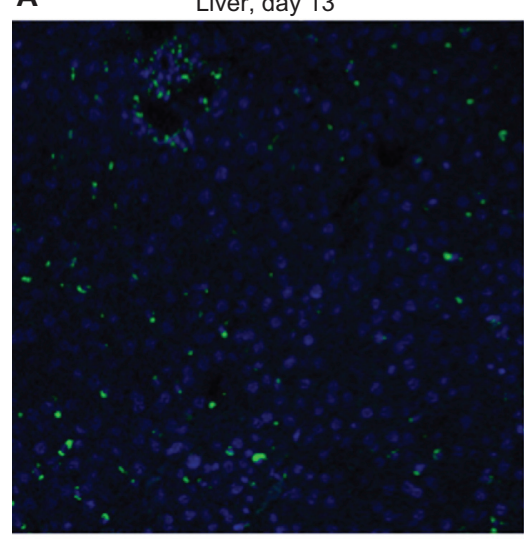

C

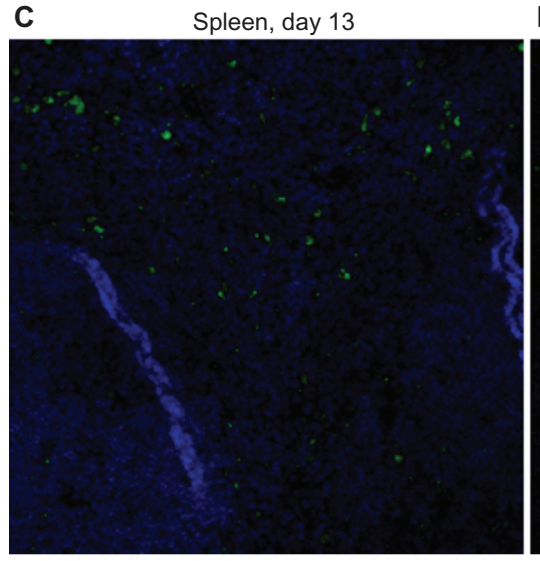

B

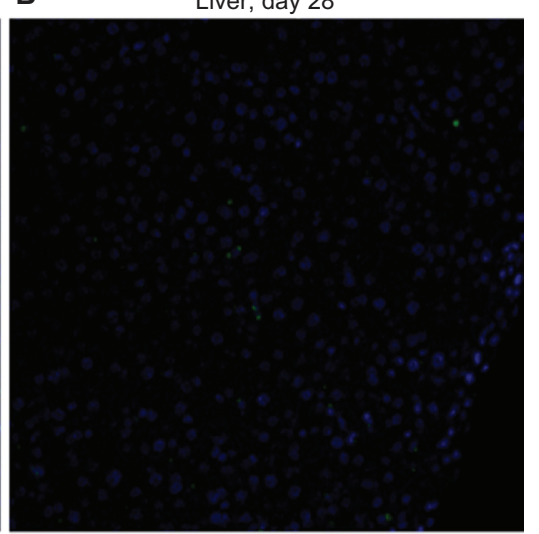

D

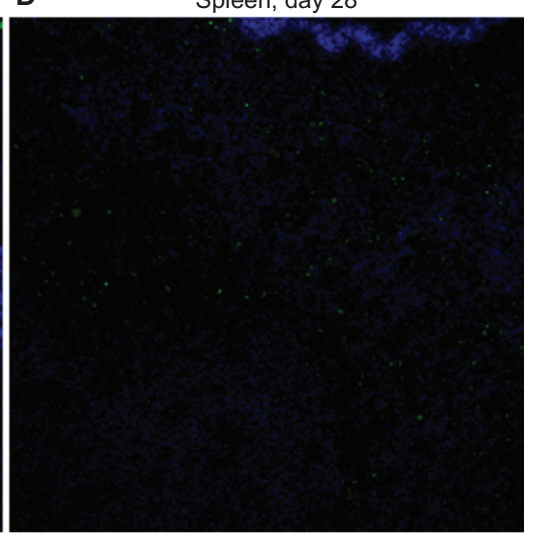

Figure 9 Immunofluorescence images (original magnification 200x) of liver and spleen tissues from toxicity analysis in female C57BI/6 mice.

Notes: Slide mounted sections $(5 \mu \mathrm{m})$ of liver (A, dayl3; B, day 28) and spleen (C, day 13; D, day 28) following administration of cisplatin-loaded cl-micelles at a cisplatin equivalent dose of $4 \mathrm{mg} / \mathrm{kg}$ were stained for PEG (anti-PEG antibody, green) and nuclei (4',6-diamidino-2-phenylindole, blue). Significant decrease in PEG staining in day 28 tissues was seen relative to corresponding day 13 tissues. Treatment was administered by bolus intravenous tail vein injection $(n=5)$, four treatments in total, with each treatment at a 4-day interval. The animals were sacrificed using $\mathrm{CO}_{2}$ euthanasia on day 13 and day 28 for toxicity analysis.

Abbreviation: PEG, poly(ethylene glycol).

sections from day 28 toxicity analysis relative to respective day 13 tissue sections (Figure 9). This might also explain the lack of toxicity to the hepatocytes or spleen since cisplatin/ cl-micelles are sequestered by the macrophages.

In spleen, the micelles were localized predominantly in the red pulp with little to no staining in the lymphoid region. Free cisplatin treatment has previously been reported to have a noteworthy effect on immunological function due to its ability to reduce lymphocytes in the thymus and the spleen, ${ }^{30}$ with changes in splenic white pulp being the most significant. ${ }^{31}$ Indeed, analysis of tissue weights at the end of the toxicity study indicated a significant decrease in overall spleen weight and the spleen to body weight ratio in the cisplatin-treated group, whereas cisplatin/cl-micelle-treated animals had normal spleen weights (Table 5). Absence of cisplatin/cl-micelles from lymphoid regions of white pulp may thus limit free-drug exposure to these follicles. Given the role of a healthy immune system in cancer growth and treatment, these results may be of significance but require further investigation.

Prolonged circulation of cisplatin incorporated into cl-micelles also facilitated substantial tumor targeting and led to increased drug level in the tumor relative to free cisplatin treatment. Since our ICP-MS method for platinum measurement did not differentiate between micellar and nonmicellar platinum, the observed increased tumor platinum levels and tumor platinum AUC likely represents the sum of both the released and polymer-bound drug. Although our previous work has shown rapid and selective cellular uptake of these micelles in vitro by neoplastic cells, ${ }^{24}$ it can still be envisaged that the cisplatin/cl-micelles partially reside in the tumor extracellular space. This compartment can serve as an additional depot from which platinum complexes are slowly released and can be taken up by the surrounding tumor cells. 
Overall, the sequestration of cisplatin/cl-micelles in tumor tissue followed by prolonged release of the active platinum species appears to translate into an enhanced anticancer effect of this micellar drug formulation. Indeed, a greater suppression of tumor growth and increased lifespan of the animals were observed during the post-treatment period in cisplatin/cl-micelle group. The extent of tumor inhibition relative to free cisplatin observed in this study is comparable or better than what has been reported for other micellar and nanoparticulate systems of similar size and charge, despite a considerably more aggressive tumor model employed in our investigations. In particular, the tumor size increased by 30-35-fold in 14 days in the control groups of animals bearing A2780 xenografts. In contrast, a subcutaneous KB epidermal carcinoma tumor model used in the study by Xue et $\mathrm{al}^{32}$ was characterized by significantly slower growth rate (the tumor size in control animals increased only by approximately 14-fold in 15 days). Yet, no improvement in antitumor activity of $\sim 110$ nm PEG-poly (N-amino acidyl)-aspartamidecisplatin micelles relative to free cisplatin was reported. Recent work by Paraskar et al $^{33}$ also utilized aggressive cancer models (subcutaneous Lewis lung carcinoma and 4T1 breast cancer xenografts) to evaluate the antitumor activity of 80-140 nm cisplatin-loaded poly-isobutylene-maleic acid nanoparticles. However, no improvement in antitumor activity relative to equidose free cisplatin was observed. In this regard, carrier size may be a critical parameter directly affecting the enhanced permeability and retention-mediated tumor accumulation, tumor penetration and, hence, the antitumor activity of a drug delivery system. A recent report by Kataoka and colleagues ${ }^{34}$ indicates that while the widely used $\sim 100 \mathrm{~nm}$ drug-containing polymeric carriers are effective in treating highly permeable tumors, smaller sized particles may be needed to improve the therapeutic performance against tumors with poor permeability. In view of these reported data, further optimization and evaluation of our formulations are necessary, including testing tumor permeability. Studies investigating the fabrication of sub$100 \mathrm{~nm}$ cl-micelles and a comprehensive analysis of the influence of the size of such materials on their drug delivery properties are underway. These structure-activity relationship data will greatly benefit design of future nanomaterials for effective platinum drug delivery.

\section{Conclusion}

This study evaluated cisplatin-loaded cross-linked polymer micelles in mice and demonstrated the improved therapeutic potential of cisplatin upon incorporation into the micelles. Cisplatin-loaded cl-micelles exhibited significantly prolonged blood circulation, higher tumor accumulation, and reduced kidney exposure relative to the free drug. As a result, the micellar cisplatin formulation showed an improved antitumor activity in a mouse ovarian tumor xenograft model without any indication of systemic toxicity. Thus, our findings of markedly reduced toxicity of the cl-micelle-bound cisplatin in combination with improved pharmacokinetics and enhanced antitumor efficacy indicate that cisplatin/ cl-micelles may have considerable benefits in clinical applications as an effective treatment modality in cancer and warrant further optimization for delivery of platinum-containing therapeutics.

\section{Acknowledgments}

We thank the Flow Cytometry Research Core (Dr Charles A Kuszynski) and Confocal Microscopy Core facilities (University of Nebraska Medical Center) for their assistance in these studies. This work was supported by grants from USA National Institutes of Health CA116590 (TKB). We acknowledge the assistance of the Nanomaterials Core facility of the Center for Biomedical Research Excellence (CoBRE), Nebraska Center for Nanomedicine supported by NIH grant 1P20RR021937. The purchase of the Varian ICP-MS at the University of Nebraska at Omaha was supported by USA NSF grant 0411164 (to FCL and others). Additional support for the maintenance and repair of the instrument was provided by the University of Nebraska at Omaha, Chemistry Department.

\section{Disclosure}

The authors declare no conflicts of interest in this work.

\section{References}

1. Gordon M, Hollander S. Review of platinum anticancer compounds. J Med. 1993;24(4-5):209-265.

2. Kelland L. The resurgence of platinum-based cancer chemotherapy. Nat Rev Cancer. 2007;7(8):573-584.

3. Cvitkovic E. Cumulative toxicities from cisplatin therapy and current cytoprotective measures. Cancer Treat Rev. 1998;24(4): 265-281.

4. Hartmann JT, Lipp HP. Toxicity of platinum compounds. Expert Opin Pharmacother. 2003;4(6):889-901.

5. Calvert H, Judson I, van der Vijgh WJF. Platinum complexes in cancer medicine - pharmacokinetics and pharmacodynamics in relation to toxicity and therapeutic activity. Cancer Surv. 1993;17: 189-217.

6. Stewart DJ. Mechanisms of resistance to cisplatin and carboplatin. Crit Rev Oncol Hematol. 2007;63(1):12-31.

7. Haxton KJ, Burt HM. Polymeric drug delivery of platinum-based anticancer agents. J Pharm Sci. 2009;98(7):2299-2316. 
8. Harper BW, Krause-Heuer AM, Grant MP, Manohar M, GarbutcheonSingh KB, Aldrich-Wright JR. Advances in platinum chemotherapeutics. Chemistry. 2010;16(24):7064-7077.

9. Bontha S, Kabanov AV, Bronich TK. Polymer micelles with crosslinked ionic cores for delivery of anticancer drugs. J Control Release. 2006;114(2):163-174.

10. Nishiyama N, Yokoyama M, Aoyagi T, Okano T, Sakurai Y, Kataoka K. Preparation and characterization of self-assembled polymer-metal complex micelle from cis-dichlorodiammineplatinum(II) and poly(ethylene glycol)-poly(alpha, beta-aspartic acid) block copolymer in an aqueous medium. Langmuir. 1999;15(2):377-383.

11. Allen C, Maysinger D, Eisenberg A. Nano-engineering block copolymer aggregates for drug delivery. Colloids Surf B Biointerfaces. 1999;16(1-4):3-27.

12. Rosler A, Vandermeulen GWM, Klok HA. Advanced drug delivery devices via self-assembly of amphiphilic block copolymers. Adv Drug Deliv Rev. 2001;53(1):95-108.

13. Nukolova NV, Oberoi HS, Cohen SM, Kabanov AV, Bronich TK. Folatedecorated nanogels for targeted therapy of ovarian cancer. Biomaterials. 2011;32(23):5417-5426.

14. Oberoi HS, Laquer FC, Marky LA, Kabanov AV, Bronich TK. Core cross-linked block ionomer micelles as $\mathrm{pH}$-responsive carriers for cis-diamminedichloroplatinum(II). J Control Release. 2011;153(1): 64-72.

15. Kim JO, Kabanov AV, Bronich TK. Polymer micelles with cross-linked polyanion core for delivery of a cationic drug doxorubicin. J Control Release. 2009;20:197-204.

16. Kim JO, Sahay G, Kabanov AV, Bronich TK. Polymeric micelles with ionic cores containing biodegradable cross-links for delivery of chemotherapeutic agents. Biomacromolecules. 2010;11(4):919-926.

17. Bronich TK, Keifer PA, Shlyakhtenko LS, Kabanov AV. Polymer micelle with cross-linked ionic core. J Amer Chem Soc. 2005;127(23): 8236-8237.

18. King FG, Dedrick RL. Physiological pharmacokinetic parameters for cis-dichlorodiammineplatinum(II) (DDP) in the mouse. J Pharmacokinet Biopharm. 1992;20(1):95-99.

19. Gaymalov ZZ, Yang ZZ, Pisarev VM, Alakhov VY, Kabanov AV. The effect of the nonionic block copolymer pluronic P85 on gene expression in mouse muscle and antigen-presenting cells. Biomaterials. 2009;30(6):1232-1245.

20. McIntosh DP, Cooke RJ, McLachlan AJ, Daley-Yates PT, Rowland M. Pharmacokinetics and tissue distribution of cisplatin and conjugates of cisplatin with carboxymethyldextran and A5B7 monoclonal antibody in CD1 mice. J Pharm Sci. 1997;86(12):1478-1483.

21. Newman MS, Colbern GT, Working PK, Engbers C, Amantea MA Comparative pharmacokinetics, tissue distribution, and therapeutic effectiveness of cisplatin encapsulated in long-circulating, pegylated liposomes (SPI-077) in tumor-bearing mice. Cancer Chemother Pharmacol. 1999;43(1):1-7.
22. Go RS, Adjei AA. Review of the comparative pharmacology and clinical activity of cisplatin and carboplatin. J Clin Oncol. 1999;17(1): 409-422.

23. Kim JO, Nukolova NV, Oberoi HS, Kabanov AV, Bronich TK. Block ionomer complex micelles with cross-linked cores for drug delivery. Polym Sci Ser A Chem Phys. 2009;51(6):708-718.

24. Sahay G, Kim JO, Kabanov AV, Bronich TK. The exploitation of differential endocytic pathways in normal and tumor cells in the selective targeting of nanoparticulate chemotherapeutic agents. Biomaterials. 2010;31(5):923-933.

25. Levi FA, Hrushesky WJ, Halberg F, Langevin TR, Haus E, Kennedy BJ. Lethal nephrotoxicity and hematologic toxicity of cisdiamminedichloroplatinum ameliorated by optimal circadian timing and hydration. Eur J Cancer Clin Oncol. 1982;18(5):471-477.

26. Duncan R. The dawning era of polymer therapeutics. Nat Rev Drug Discov. 2003;2(5):347-360.

27. Waser PG, Muller U, Kreuter J, et al. Localization of colloidal particles (liposomes, hexylcyanoacrylate nanoparticles and albumin nanoparticles) by histology and autoradiography in mice. Int J Pharm. 1987;39(3):213-227.

28. Souris JS, Lee $\mathrm{CH}$, Cheng $\mathrm{SH}$, et al. Surface charge-mediated rapid hepatobiliary excretion of mesoporous silica nanoparticles. Biomaterials. 2010;31(21):5564-5574.

29. Cho M, Cho WS, Choi M, et al. The impact of size on tissue distribution and elimination by single intravenous injection of silica nanoparticles. Toxicol Lett. 28 2009;189(3):177-183.

30. Basinger MA, Jones MM, Holscher MA. L-methionine suppresses pathological sequelae of cis-platinum in the rat. Fundam Appl Toxicol. 1990;14(3):568-577.

31. Milicevic Z, Slepcevic V, Nikolic D, Zivanovic V, Milicevic NM. Effects of cis-diamminedichloroplatinum-II (cisplatin) on the splenic tissue of rats - a histoquantitative study. Exp Mol Pathol. 1994;61(2): 77-81.

32. Xue YK, Tang XX, Huang J, et al. Anti-tumor efficacy of polymerplatinum(II) complex micelles fabricated from folate conjugated PEG-graft-alpha,beta-poly [(N-amino acidyl)-aspartamide] and cis-dichlorodiammine platinum(II) in tumor-bearing mice. Colloids Surf B Biointerfaces. 2011;85(2):280-288.

33. Paraskar AS, Soni S, Chin KT, et al. Harnessing structure-activity relationship to engineer a cisplatin nanoparticle for enhanced antitumor efficacy. Proc Natl Acad Sci U S A. 2010;107(28):12435-12440.

34. Cabral H, Matsumoto Y, Mizuno K, et al. Accumulation of sub-100 nm polymeric micelles in poorly permeable tumours depends on size. Nat Nanotechnol. 2011;6(12):815-823.
International Journal of Nanomedicine

\section{Publish your work in this journal}

The International Journal of Nanomedicine is an international, peerreviewed journal focusing on the application of nanotechnology in diagnostics, therapeutics, and drug delivery systems throughout the biomedical field. This journal is indexed on PubMed Central,

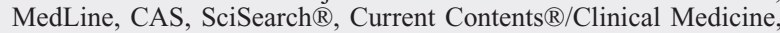

\section{Dovepress}

Journal Citation Reports/Science Edition, EMBase, Scopus and the Elsevier Bibliographic databases. The manuscript management system is completely online and includes a very quick and fair peer-review system, which is all easy to use. Visit http://www.dovepress.com/ testimonials.php to read real quotes from published authors. 